\title{
An unusual surgical success with a defective penile prosthesis
}

\author{
Chen Zhao, MD; Bo Ram Choi, MD; ${ }^{*}$ Young Beom Jeong, MD; Jong Kwan Park, MD; ;
}

\begin{abstract}
*Department of Urology, Medical School, Jeonju, Korea; ${ }^{\dagger}$ Institute for Medical Sciences, Chonbuk National University and Research Institute, Jeoniu, Korea; ₹Clinical Trial Center of Clinical Medicine of
\end{abstract} Chonbuk National University Hospital, Jeoniu, Korea

Cite as: Can Urol Assoc J 2011;5(6):E1 16-E1 18; http://dx.doi.org/10.5489/cuaj.10162

\section{Abstract}

A defective penile prosthesis is disconcerting for the surgeon performing an implantation in a patient with erectile dysfunction. We became aware of the defective Titan penile prosthesis just after insertion of the left cylinder, which had an abnormal rotation (180 degrees) due to abnormal rotated tubing, which occurred at the manufacturing plant. We successfully performed the implantation by cutting and re-connecting the tubing between the cylinder and pump with a spare connector. When the surgeon encounters a defective penile prosthesis with abnormal rotation of the cylinder due to abnormal rotated tubing in the operative field, disconnection and re-connection of the tubing using an extra connector are alternative cost-effective maneuvers.

\section{Introduction}

Implantation of a three-piece inflatable penile prosthesis (TPIPP) is a standard surgical procedure. ${ }^{1}$ Previously, we reported a severe complication, urethral stricture caused by compression, which occurred by a twisted tubing resulting from abnormal rotation of the cylinder. ${ }^{2,3}$ The tubing between the cylinder and the pump had been connected at the manufacturing plant, which was an unexpected situation for the surgeon. These defects are typically discovered incidentally just before or after insertion of the cylinder.

This is the first report of an unusual experience in the management of a defective penile prosthesis, which was identified just after insertion of the cylinder.

\section{Case report}

A 72-year-old male presented with a malfunction of the three pieces of an inflatable penile prosthesis (TPIPP), which had been implanted. Thirteen years earlier, he underwent implantation using an inflatable penile prosthesis Mentor
Alpha I (Mentor Corp., Santa Barbara, CA) via the scrotal approach. The malfunction of the prosthesis had developed from 3 months before visit. During the physical examination, pumping did not inflate both cylinders.

Two weeks later, we performed a re-implantation of the TPIPP Titan (Coloplast Corp., Minneapolis, MN). A 16 French Foley catheter was inserted into the urethra, then the old TPIPP was successfully removed and a new prosthesis was prepared for implantation. After insertion of both cylinders, we found that the tubing on the left between the pump and cylinder had rotated abnormally, resulting in compression of the urethra. The pump was also rotated 90 degrees to the left. Therefore, we removed the cylinder from the left cavernosum and noted that the left cylinder was defective with an abnormal rotation 180 degrees; this defect had occurred during the manufacturing process (Fig. 1). We replaced the cylinder into the cavernosal space after the tubing was cut and re-connected with an extra connector, resulting in a natural position of the pump (Fig. 2). The new pump was placed within the scrotum in a routine manner. Intra-operative antibiotic irrigation (gentamycin and vancomycin) was performed. A closed suction drain was placed in the scrotum. The dartos fascial layer was closed in 2 layers with a running 3-0 absorbable suture. The subcutaneous tissues were closed with interrupted 4-0 absorbable sutures. The skin incisions were closed with 3-0 monofilament sutures. One gram of vancomycin and $100 \mathrm{mg}$ of isepamicin were administered to the patient every 12 hours beginning 1 hour preoperatively and continued for 2 days. The perioperative antibiotics were replaced by oral ciprofloxacin, metronidazole and rifampin for an additional 5 days. The drain was removed on postoperative day 1 . An indwelling urethral catheter was left in place for 1 day postoperatively. The patient was discharged on day 3 postoperatively with a 21-day course of ciprofloxacin and rifampin. The TPIPP was functioning satisfactorily at the 12-month follow-up without any complications or voiding difficulties. 


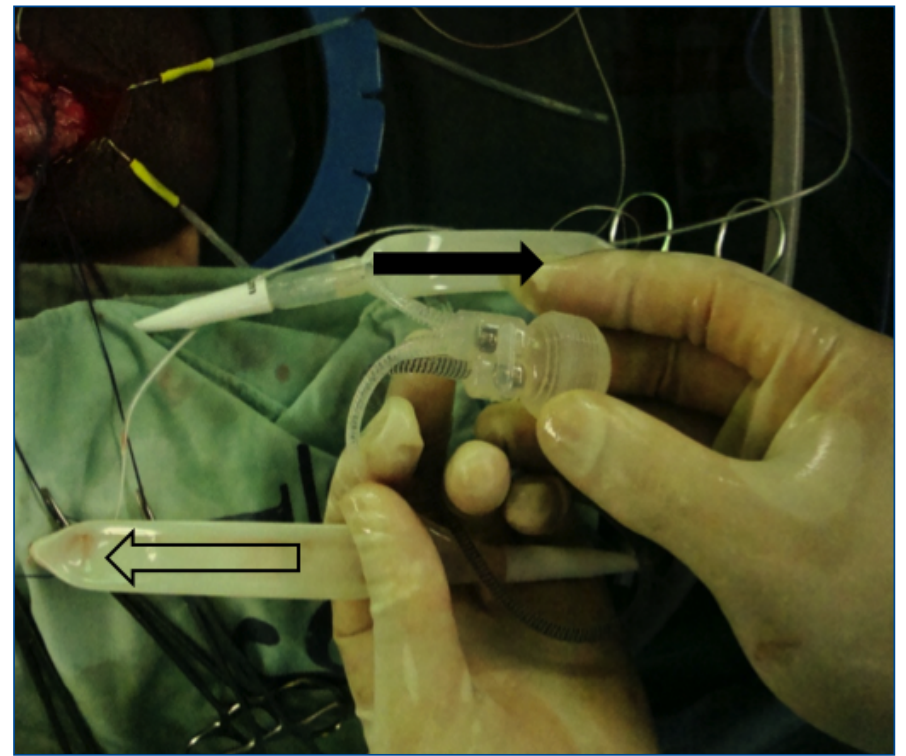

Fig. 1. The left cylinder was abnormally rotated 180 degrees (solid black arrow) in relation to the longitudinal axis of the right cylinder (open black arrow) with twisting of the left tubing, which had been originating from the manufacturing plant.

\section{Discussion}

The implantation of a penile prosthesis is a safe and standard solution for the patient with erectile dysfunction who has not been completely cured with medical treatment. Of these patients, $70 \%$ to $80 \%$ who undergo penile prosthesis implantations are satisfied with the results. Nevertheless, the mechanical failure rate is $<5 \%$ in the first year and $17.4 \%$ to $50 \%$ at 10 years; infection rates remain fairly constant over the years at about $3 \% .^{3-5}$

In our experience, insertion of an abnormally rotated cylinder twists the connecting tubing between the cylinder and the pump, then the twisted tubing compresses the urethra, resulting in severe urethral stricture..$^{2,3}$ Therefore, insertion of an abnormally rotated cylinder affects the fate of the prosthesis.

During implantation of a penile prosthesis, limiting operating room traffic, using sterile techniques, causing minimal tissue trauma, reducing the operating time and effective wound closure are critical factors in preventing infections..$^{6,7}$ However, inappropriate preparation of the instruments or a defective penile prosthesis may increase the operative time, resulting in frustration for the surgeon. We are usually unaware of a defective penile prosthesis before insertion of the cylinder.

In our case, the twisted tubing was noted just after insertion of the cylinder. We thought the twisted tubing was caused by abnormal rotation of the cylinder. However, the abnormal rotation of the cylinder (180 degrees) was secondary to the defective tubing, which occurred at the manufacturing

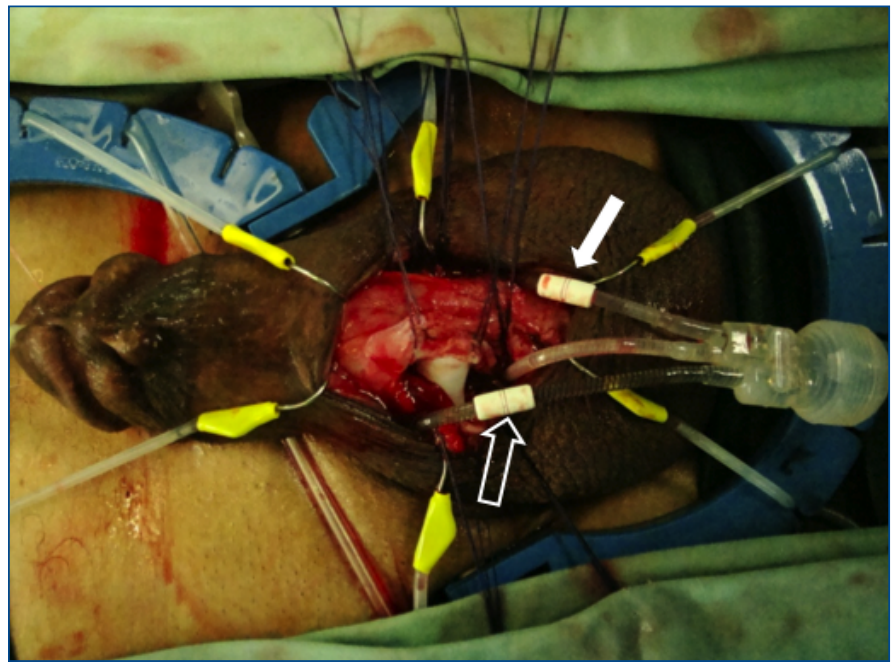

Fig. 2. Operative view shows neutral locations of the tubing, pump, and cylinders. The left tubing was cut and re-connected with an extra connector (white solid arrow) resulting in a natural position of the pump. The white open arrow shows the connector between the pump and reservoir.

plant. We did not have any additional prostheses in the operating room. A spare connector helped correct the defect. To overcome the abnormal situation with the tubing, we cut the tubing and re-connected the end using the spare connector. As a result, the tubing twisted was normalized and the cylinder resumed a normal direction.

\section{Conclusion}

The surgeon must confirm any mechanical abnormalities of inflatable penile prostheses before insertion of the cylinder to achieve a safe implantation. Despite the prolonged operative time required to implant a defective prosthesis, the defect should be corrected in the operating room before completing the surgery.

Competing interests: None declared.

Acknowledgements: This study was supported by grants of Clinical Trial Center Medical Device of Chonbuk National University Hospita and of the Korea Healthcare technology R\&D Project, Ministry for Health, Welfare \&Family Affairs, Republic of Korea (A091220).

This paper has been peer-reviewed.

\section{References}

1. Wilson SK, Delk JR, Salem EA, et al. Long-term survival of inflatable penile prostheses: single surgical group experience with 2,384 first-ime implants spanning two decades. I Sex Med 2007;4:1074-9.

2. Cui WS, Kim SD, Choi KS, et al. An unusual success with simultaneous urethral repair and reimplantation of penile prosthesis in a patient with urethral stricture induced by rotated tubing. J Sex Med 2009;6:1783-6. 
Zhao et al.

3. Lee SW, Park BH, Lim JH, et al. Surgical TIPS to prevent urethral stricture during insertion of an inflatable penile prosthesis. Int I Urol 2007;15:162-5.

4. Wilson SK, Delk JR, Salem EA, et al. Long-term survival of inflatable penile prostheses: single surgical group experience with 2,384 first-time implants spanning two decades. I Sex Med 2007;4:1074-9.

5. Minervini A, Ralph DJ, Pryor JP. Outcome of penile prosthesis implantation for treating erectile dysfunction: experience with 504 procedures. BJU Int 2005;97:129-33.

6. Jarow JP. Risk factors for penile prosthetic infection. J Urol 1996; 156:402-4.
7. Carson CC. Diagnosis, treatment and prevention of penile prosthesis infection. Int I Impot Res 2003;15(Suppl 5):139-46.

Correspondence: Dr. Jong Kwan Park, Department of Urology, Chonbuk National University, Medical School, 2-20, Keum-Am-Dong-San, Chonju 560-180, South Korea; fax: 82-63-250-1564; rain@ chonbuk.ac.kr 